\title{
Estrategia de desarrollo rural, una valoración desde las perspectivas endógenas en el caso de la producción de sombreros de paja toquilla, Sigsig-Azuay
}

Rural development strategy, an assessment from endogenous perspectives in the case of toquilla straw hat production, Sigsig-Azuay.

Juan José Uyaguari Quezada. ${ }^{1}$, Dorian Damián Flores Aguilera. ${ }^{2} \&$ Yonimiler Castillo Ortega. ${ }^{3}$

\section{Abstract. $\quad$ DOI: https://doi.org/10.33262/concienciadigital.v4i1.2.1578}

Introduction. Local development refers to the generation of coordination and organization spaces between the actors of a territory, seeking the deployment and performance of determining factors through an adequate operation whose objective is to achieve the well-being of its inhabitants. Objective. Faced with this, this research seeks to contribute with the design of a development strategy that stimulates the manufacture of hats, considering political-institutional aspects, of productive development and training. Methodology. The research is based on the collection of primary information. On the one side, in-depth interviews with influential actors in the artisanal sector and, on

\footnotetext{
${ }^{1}$ Universidad Católica de Cuenca, Posgrado, Maestría en Desarrollo Local Mención en Ordenamiento Territorial, Cuenca, Ecuador, juan.uyaguari@est.ucacue.edu.ec, https://orcid.org/0000-0001-8382-5742 ${ }^{2}$ Universidad Católica de Cuenca, Carrera de economía, Cuenca, Ecuador, dorian.flores@ucacue.edu.ec, https://orcid.org/0000-0003-0762-8928

${ }^{3}$ Universidad Católica de Cuenca, Posgrado, Maestría en Desarrollo Local Mención en Ordenamiento Territorial, Cuenca, Ecuador, ycastilloo@ucacue.edu.ec, https://orcid.org/0000-0002-7710-5199
} 
the other hand, surveys of producers of toquilla straw hats. For the application of the survey, a simple random sampling has been carried out. Result. The weaving of toquilla straw hats is very relevant to the local economy, especially women in rural areas of the cantonal head and in the parishes of Cuchil, Guel, San Bartolomé and Ludo. This activity represents the most important income for each of them. In this sense, the strategy proposes to generate market opportunities, diversify and expand sales channels, improve models and improve the quality of the hat. Conclusion. The manufacture of hats involves various activities, actors and factors, which pose important challenges when it comes to inserting itself as a sector in the local economic development of the canton, ranging from the search for a balance between production costs versus the sale price, the demand for the product in the market, its commercialization and institutional strengthening for its promotion. However, it can be seen that there is progress in the associations that have diversified the products, which comes hand in hand with training and institutional promotion processes for their sustainability.

Keywords: Rural development, development strategy, toquilla straw hat, crafts

\section{Resumen.}

Introducción. El desarrollo local hace referencia a la generación de espacios de coordinación y organización entre los actores de un territorio procurando el despliegue y desempeño de factores determinantes mediante un funcionamiento adecuado cuyo objetivo es alcanzar el bienestar de sus habitantes. Objetivo. Frente a ello, esta investigación procura aportar con el diseño de una estrategia de desarrollo que estimule la fabricación de sombreros considerando aspectos político-institucionales, de fomento productivo y de capacitación. Metodología. La investigación se fundamenta en la recopilación de información primaria. Por un lado, entrevistas a profundidad a los actores influyentes en el sector artesanal y por otro lado la realización de encuestas a los productores de sombreros de paja toquilla. Para la aplicación de la encuesta se ha ejecutado un muestreo aleatorio simple. Resultado. El tejido de sombreros de paja toquilla es muy relevante para la economía local, especialmente las mujeres de las zonas rurales de la cabecera cantonal y de las parroquias de Cuchil, Guel, San Bartolomé y Ludo. Esta actividad representa el ingreso más importante para cada una de ellas. En este sentido la estrategia propone generar oportunidades de mercado, diversificar y ampliar los canales de venta, mejorar los modelos y mejorar la calidad del sombrero. Conclusión. La fabricación de sombreros involucra diversas actividades, actores y factores, que plantean retos importantes a la hora de insertarse como sector en el desarrollo económico local del cantón, que van desde la búsqueda de un equilibrio entre los costos de elaboración versus el precio de venta, la demanda del producto en el mercado, su comercialización y de fortalecimiento institucional para su promoción. Sin embargo, se puede ver que hay avances en las asociaciones que han diversificado los productos, lo 
cual viene de la mano con procesos de capacitación y de fomento institucional para su sostenibilidad.

Palabras claves: Desarrollo rural, estrategia de desarrollo, sombrero de paja toquilla, artesanía.

\section{Introducción.}

\section{El desarrollo económico rural: aspectos teóricos}

En los países de America Latina se empieza a evidenciar el desarrollo económico como consecuencia de reformas constitucionales cuyo objetivo fue dar mayor preponderancia a las políticas macroeconómicas sobre las políticas sectoriales. A la suma de estos acontecimientos se lo denominó como Consenso de Washington (Schejtman, 2010).

No obstante, han hecho presencia otros enfoques y modelos de desarrollo, como el de desarrollo económico local planteado por Albuquerque (2004) que lo considera como "un proceso de desarrollo participativo que fomenta los acuerdos de colaboración entre los principales actores públicos y privados de un territorio, posibilitando el diseño y la puesta en práctica de una estrategia de desarrollo común”. O el de Vázquez Barquero (1988) que señala que es "un proceso de crecimiento y cambio estructural que mediante la utilización del potencial de desarrollo existente en el territorio conduce a la mejora del bienestar de la población de una localidad o una región". Si bien las concepciones son diferentes, los dos autores ponen en el centro de su análisis a la población.

Por otro lado, Rojas (2009) para el autor el concepto que mejor se acondiciona a esta investigación, para quien el "Desarrollo Local debe generar un mejor despliegue y desempeño de factores determinantes mediante un funcionamiento adecuado y la generación de espacios de coordinación y organización entre los actores del sistema económico local con el fin de alcanzar el bienestar de ésta población”.

Para Durston (2002) en "América Latina se ha puesto en el centro de la agenda pública una segunda ola de reformas de las políticas sociales, en la cual la sociedad civil está llamada a servir de contrapeso para corregir las fallas del mercado en la entrega de los servicios". En tanto que Aghón (et al, 2001) considera que "la visión del desarrollo económico local da prioridad a las microempresas y pequeñas empresas, a causa de la alta proporción que representan dentro de las empresas latinoamericanas y su importancia para el empleo y la generación de renta".

Este trabajo centra su atención en el ser humano como eje central del desarrollo.

Para esta investigación es relevante el desarrollo rural abordado por Kayser, que determina como sus características: territorios con baja densidad poblacional, uso de suelo con predomino de actividades agropecuarias, habitantes con fuertes lazos sociales y de 
conocimiento personal. (Citado por Cortés, 2019, p.2). Y lo señalado por Bardhan (2001) que añade que los espacios rurales muestran a pocos habitantes que acaparan gran cantidad de recursos y que por lo tanto inciden directamente en las decisiones políticas de la Autoridad local. En contraposición otro conglomerado más amplio de habitantes que viven en espacios más dispersos con cobertura de servicios deficitarios que contribuyen a situaciones mayores de pobreza y con amplias dificultades para ejecutar proyectos conjuntos de desarrollo.

La problemática central plantea solucionar las particularidades que generan las políticas aplicadas sobre las principales actividades económicas que a priori se ejecutan de manera homogénea bajo la concepción que los actores de mercado tienen un mismo actuar e intereses, (Nathalie et al.,2010). "Esto vuelve a plantear la cuestión de dónde es más eficaz el dinero público (Ceña, 1995) si se desea diversificar la estructura productiva de los espacios rurales atrasados: en la inversión productiva, en la inversión en infraestructuras y equipamientos o en los subsidios personales, y, si éstos últimos sirven para mejorar la vida en el medio rural o para mantener las diferencias de renta" (Gómez 2011).

Con relación al tema de esta investigación, entre los aspectos positivos más relevantes del desarrollo económico en el sector artesanal se destacan los planteados por (Nathalie et al., 2010) que "resalta el proceso complejo de construcción histórico-social que comprende las dimensiones patrimoniales, culturales, sociales, saberes, acervo tecnológico, entre otros activos tangibles e intangibles territoriales"; lo señalado por (Palmitesta 2005) que añade que "lo rural cumple un rol fundamental para la supervivencia de la cultura..."; y, finalmente (Schejtman,2010) quien considera que "el empleo rural no agrícola, permite estabilizar los ingresos compensando la estacionalidad de la producción así como diversificar las fuentes de ingreso reduciendo los efectos de los riesgos inherentes a la agricultura".

Igualmente, y de acuerdo a (Mora \& Sumpsi, 2004) para alcanzar el desarrollo rural eficaz y que muestre efectos en la economía local garantizando su sustentabilidad será necesario destinar esfuerzos que vayan más allá de la construcción de equipamientos e infraestructura básica como son las vías, sistemas de agua, bodegas etc. Añade que no se considera de manera integral programas de desarrollo para esta población. Los esfuerzos se destinan particularmente proyectos específicos como el fortalecimiento institucional ó el fomento productivo, construcción de equipamientos o programas sociales que por sí solos no cumplen los objetivos de desarrollo.

Para (Rojas, 2009) el desarrollo económico local "requiere de una alta dosis de preparación de los actores locales". Este postulado se lo debe incorporar como una estrategia transversal al proceso transformador de desarrollo que debe contemplar procesos de aprendizaje conjuntos, intercambio de experiencias, grupos de trabajo e 
investigación y desarrollo con el objetivo de mantener capacitación permanente y de mejorar integralmente sus competencias.

Para (Alburquerque, 1997) lo que se requiere implementar es "un sistema económico local que esté constituido por el conjunto de actores productivos y de relaciones económicas y socio-laborales en torno a un rubro de la economía, que tienen un cierto grado de coherencia socio-económica y territorial, no necesariamente coincidente con la división administrativa del territorio".

En este contexto, en el siguiente acápite se presenta con un mayor nivel de detalle, información referente a los componentes de la estrategia de desarrollo económico local y como éstas pueden transformar y dinamizar las actividades económicas.

\section{Componentes para una estrategia de desarrollo local}

Para Vázquez Barquero (2000) el planteamiento y cumplimiento de una estrategia de desarrollo rural es el camino a seguir para alcanzar el desarrollo local cuyas bases se fundamentan en cuatro ejes que son la competitividad, la productividad, la equidad y la ecología.

Sin embargo, de lo antes mencionado Vázquez Barquero (2000) añade que cada territorio tiene particularidades y que por lo tanto requiere de la aplicación de instrumentos específicos para solventar sus necesidades. Consecuentemente, según el mismo autor "En los territorios con baja densidad de población, con población envejecida y donde los recursos naturales y el patrimonio artístico y cultural se deteriora progresivamente, las únicas acciones posibles son las subvenciones y el apoyo de las administraciones públicas orientadas a mantener la población y los valores medioambientales y culturales".

En contraposición, para Acampora (2007) la formulación de una estrategia exitosa está en relación directa con la creación de instituciones locales, así como la formulación de ordenanzas y reglamentos que se adapten a las necesidades territoriales y precautelen los beneficios e intereses de los involucrados.

Rojas (2009) refuerza el concepto considerando que "La identidad territorial puede ser el centro de toda estrategia territorial. Un territorio que sale del anonimato a partir de sus potencialidades tiene un cambio notable en la forma en que es percibido: su imagen le da fuerza de atracción por lo que sus productos son más solicitados".

También es importante destacar que, en "Las áreas rurales más desfavorecidas, la cuestión reside en que las debilidades estructurales son tan importantes y las potencialidades locales tan limitadas, que se reduce, drásticamente, el margen de maniobra posible de la estrategia en la elección de las actividades a partir de las que se motoriza el desarrollo; no 
obstante, la estrategia de desarrollo endógeno es conseguir generar las externalidades, que permitan rendimientos crecientes en los sistemas productivos locales y, por ende, el desarrollo económico del territorio”. (Vázquez Barquero, 2000).

Encaminar estrategias hacia el desarrollo económico rural en la población artesanal no es tarea fácil. Sin embargo, por medio del campo teórico se muestran múltiples líneas de acción que van desde lo general hacia lo particular considerando aspectos políticoinstitucionales, de fomento productivo a través de la capacitación y formación, así como lo relacionado estrictamente con el ser humano, que para el autor es el eje central del estudio. A continuación, se detallan las estrategias de las principales corrientes teóricas relacionadas con el tema.

1. "Las estrategias y políticas de desarrollo rural deben impulsar procesos sociales y económicos de largo período de maduración. La lógica dominante de las intervenciones de las agencias y gobiernos, limitadas en el tiempo y en el espacio, no contribuye al éxito de los programas de desarrollo rural, por lo que. con proyectos desarticulados, dispersos en el espacio y limitados en el tiempo no se logrará el desarrollo de las áreas rurales," (Mora \& Sumpsi, 2004).

2. Moisá et al., (2010) considera que "Se debe brindar asistencia técnica por parte de las instituciones de educación superior o escuelas politécnicas, en el proceso de desarrollo rural prestando su acompañamiento a lo largo de todo el proceso productivo y en el largo plazo".

3. Los productos típicos certificados a más de ser una importante herramienta de confianza con el cliente y de protección del producto es un mecanismo de motivación de los actores locales, figurando impactos positivos en el territorio lo que puede generar nuevas propuestas de desarrollo local. Los productos típicos son particulares debido a la incorporación de los saberes y conocimientos de las generaciones, sin dejar de lado las características geográficas del territorio.

4. Schejtman (2010) considera que: los procesos de transformación productiva tienen por objeto disminuir la desigualdad y pobreza en la ruralidad, lo que conlleva al mejoramiento de procesos y productos para incorporarse en mercados más allá de sus propios territorios para la transformación productiva. La generación de plataformas público-privadas ayudará a la incorporación de los más pobres en la utilidad de la transformación productiva.

5. Para Hektor (2005) frente a los actuales mecanismos de voraz competencia la asociatividad es el mejor mecanismo para que los productores rurales puedan hacer frente a los obstáculos generados por el mercado, sino de dejar de lado aquellos instrumentos que ya existen y que pueden ser usados para alcanzar el mismo objetivo.

6. Anchundia (2016) establece que: No se puede cimentar el futuro extraño a la organización del proceso productivo, "Es el escenario donde se producen, recrean y renuevan el bienestar del artesano del sombrero; es donde se construyen, 
expresan y transforman sus representaciones del entorno en que se desenvuelve; orientado a elevar las condiciones económicas, sociales y culturales del artesano y su familia para contribuir con plenitud al progreso nacional".

7. Finalmente, "el desarrollo local como estrategia se fundamenta según B. Vachon en las relaciones entre los distintos agentes socioeconómicos y persigue los siguientes objetivos: a) Creación de un entorno adecuado que garantice el desarrollo de iniciativas por parte de la población local; b) Adaptación al contexto socioeconómico actual; c) Búsqueda de nuevas formas de desarrollo que permitan integrar los aspectos puramente económicos y los aspectos sociales, culturales y medioambientales" (Citado por Cortés, 2019, p.9).

\section{El sector artesanal de producción de sombreros de paja toquilla en el marco del desarrollo rural}

Las ciudades de la región Sierra Sur por las tendencias de evolución del Siglo XX tuvieron particulares y complejos cambios en sus dinámicas sociales, políticas y económicas, denotando importantes transformaciones en: el crecimiento urbanístico, la densificación poblacional, la implementación de servicios básicos e integración al mercado internacional para la exportación de sombreros de paja toquilla.

Los sombreros de paja toquilla, equivocadamente conocidos como Panama Hats, han sido sin duda un hito cultural de nuestro país, siendo catalogado además como un producto de gran importancia, que obtuvo el reconocimiento de la UNESCO con la declaratoria de éste como Patrimonio Intangible de la Humanidad el 05 de diciembre del año 2012, destacando el arte en la elaboración y la importancia del uso de este producto.

Según Ortiz \& López (2015) detalla que: La tradición e historia de un pueblo se hace visible en las actividades artesanales, dando como resultado las identidades locales y la diversidad cultural. Las artesanías como es el caso de los sombreros de paja toquilla tienen particularidades como el de ser parte de las actividades diarias y del trabajo de los pueblos y familias rurales; se podría manifestar que la elaboración de los sombreros de paja toquilla son la subsistencia del entorno al cual pertenece el artesano.

Autores como Martínez-Peñaloza (1982) "ven a las artesanías como formas con las que una comunidad conserva y transmite su peculiaridad física y espiritual. Constituyen un elemento importante en la indumentaria, la utilería doméstica, la simbología ceremonial, se asocian a las festividades comunitarias, representan un universo de formas, colores, texturas y materiales en que se inscribe la vida individual y colectiva de quienes lo realizan. Para este autor, es arte popular porque expresa la voluntad de ser, de un modo propio y peculiar del grupo indígena”. (Citado por Del Carpio, 2016). 
Por su parte, Pérez (2015) indica que artesanías: "se refiere al conjunto de productos u obras de arte, personalizadas, hechas por los artesanos como parte de su actividad ordinaria: obras, por tanto, habitualmente manuales, decorativas, por lo general de uso doméstico hechas con toque artístico personal”. En este mismo sentido Pérez 2015 indica que "La artesanía es parte del sistema de comunicación de un pueblo que funciona como signo y es una de las características fundamentales de la cultura. Por ello defiende que las artesanías son parte de procesos intensos de comunicación por los que se transmiten las tradiciones más íntimas de un grupo humano. Este es el sentido más profundo de las artesanías: reafirmar la tradición y asegurar la cohesión generacional" (Citado por Del Carpio, 2016, p. 82).

Al analizar lo manifestado por Ortiz \& López (2015) se destacan varias inquietudes respecto a la preservación de las tradiciones ancestrales a través de las artesanías y el cómo disminuir las condiciones de pobreza y desigualdad en las comunidades dedicadas a esta actividad; notándose que la política dictada se ha encaminado en promover la ubicación de la artesanía a nivel global promocionando el valor artesanal que lleva intrínseco las tradiciones e identidad de los productores.

Ortiz \& López, 2015, también mencionan que es importante estudiar los mercados en donde se insertará la producción de artesanías, para de esta forma identificar las condiciones de los productores con el objeto de vender sus trabajos en un amplio mercado más allá de la demanda junto con una oferta en ascenso. La tendencia del mercado tiene la característica de ser más exigente en la forma de elaboración de las artesanías, precios bajos para la comercialización y materia prima con un precio creciente.

Para finalizar es importante recalcar lo que estable Del Carpio (2016) quien menciona que las artesanías son trascendentales, sin embargo, quien la elabora es decir el artesano es aún más importante; siendo necesario enfocar los esfuerzos en el bienestar de ellos, pues, mientras haya quien elabore las artesanías estas podrán persistir en el tiempo.

\section{Definición del problema de la investigación}

En el Ecuador el "tejido de la paja toquilla forma parte de un entramado simbólico y social, porque es un elemento integrador de la comunidad familiar, de su historia y está relacionado con sus tradiciones. Gracias a esta práctica se tejieron las relaciones históricas entre la Costa y la Sierra, por el intercambio de este saber, y por la mayor fluidez en las comunicaciones que incrementaron su comercio, por el hecho de que las comunicaciones empezaron a ser más fluidas".

De acuerdo a María Aguirre (2018) en su tesis "Las tejedoras de paja toquilla de la provincia del Azuay" comenta que "Esta actividad permitió algunas transformaciones sociales, económicas y políticas a nivel nacional, en el fomento de políticas públicas 
educativas en el Azuay y Cañar, a través de las cuales esta actividad se determinó como obligatoria en la enseñanza para todos los estudiantes y la población de las clases populares en general, por el impacto en la economía local como principal rubro de exportación y en cuanto a que es parte de la actual vestimenta de las cholas".

Con estos antecedentes, la presente investigación se centra en el sector artesanal de la manufactura de sombreros en el cantón Sigsig de la Provincia del Azuay. En una breve síntesis, este cantón es uno de los quince de esta provincia y consta de seis parroquias rurales: Guel, Cuchil, San José de Raranga, Ludo, Jima, San Bartolomé y una parroquia urbana: Sigsig. Tiene una superficie aproximada de $674.53 \mathrm{Km} 2$. La producción de sombreros se realiza principalmente en la cabecera cantonal y las comunidades de su periferia, además de sus Parroquias colindantes Guel y Cuchil.

De acuerdo a información obtenida del GAD Municipal de Sigsig, uno de los problemas que se evidencia en la producción de sombreros es la falta de experiencia comercial y asociativa, que ha tenido como consecuencias el cierre de algunas asociaciones y el desconocimiento del sector sobre las demandas del mercado, lo que aparentemente llevó a su baja demanda. A ello se suma la presencia de intermediarios que condicionan la adquisición de sombreros a un precio bajo y a modelos específicos, relacionados con el tamaño, el color y el diseño, situación que limitan su demanda y disminuye el margen de utilidad para el artesano, que en este caso es quien realiza el mayor esfuerzo.

Frente a ello, esta investigación procura aportar con el diseño de una estrategia de desarrollo que estimule la fabricación de sombreros en el cantón Sigsig, acorde con la realidad del sector.

\section{Metodología}

La investigación se fundamenta en la recopilación de información primaria mediante la formulación de entrevistas semiestructuradas que se realizaron desde dos fuentes de información. Por un lado, entrevistas a profundidad a los actores influyentes en el sector artesanal en el cantón, como son las presidentas de las dos asociaciones productivas de sombreros: Asociación María Auxiliadora y Asociación Tesya, al Alcalde del Cantón y a la Directora de desarrollo económico del Municipio; y por otro lado se realizaron encuestas a los productores de sombreros de paja toquilla del cantón Sigsig de manera presencial en el domicilio de estas personas.

Para la aplicación de la encuesta se ha ejecutado un muestreo aleatorio simple y en un segundo momento un muestreo por conglomerados en consideración a que la elaboración de sombreros se realiza en cuatro parroquias dentro del cantón: Guel, Cuchil, Ludo y Sigsig. El tamaño de la población total se ha definido de acuerdo a información disponible del último censo realizado por INEC 2010 en relación a la "rama de actividad", de donde 
se ha verificado que las actividades de manufactura son las relacionadas estrictamente con la elaboración de sombreros de paja toquilla.

A partir de estos datos y con un nivel de confianza del $95 \%$, un error máximo admisible del $5 \%$ y un valor probabilidad de éxito o fracaso igual al $50 \%$, se ha calculado la muestra.

En la encuesta se han contemplado preguntas abiertas y cerradas de elección múltiple. En el caso de las preguntas abiertas ha sido necesaria aglomerar el conjunto de respuestas con alta variabilidad a fin de obtener información con un alto nivel de consolidación.

De una población total de 3022 personas dedicadas a la actividad de tejido de sombreros en Sigsig, el resultado de la muestra fue de 296 encuestas. Las mismas que fueron distribuidas: 162 en la Parroquia Sigsig, 26 en la Parroquia Cuchil, 33 en la Parroquia Guel y 75 en la Parroquia Ludo.

El cuestionario realizado fue estructurado por componentes o factores determinantes en el proceso de elaboración de sombreros, en donde se obtuvo información del perfil del productor, los tipos de sombreros elaborados, los volúmenes de producción, ventas y costos, las formas de comercialización, los niveles de asociatividad, así como sus principales problemas en proceso productivo.

Para la ejecución de la entrevista a profundidad a los principales actores, se la ejecutó conforme un cuestionario elaborado con anterioridad que planteaba realizar consultas específicas para cada actor con el fin de conocer cuál es su rol dentro de este sector económico y en qué medida desde este ámbito se puede generar una estrategia que propenda al desarrollo económico local de las y los artesanos.

Los datos obtenidos en este levantamiento de información tanto a través de la encuesta como las entrevistas han sido tabulados y sistematizados correspondientemente y cuyos resultados se muestran en el siguiente acápite.

La validación de la propuesta ha sido realizada por los funcionarios Municipales de la Dirección de Planificación estratégica, así como de la Dirección de desarrollo económico y la máxima autoridad del GAD Municipal de Sigsig.

\section{Resultados y discusión.}

Los resultados que se muestran a continuación presentan las principales características de los productores de sombreros en relación a su perfil, las características de su producción y comercialización, así como sus expectativas en relación a sus principales problemas y posibles soluciones para este sector económico. 
Perfil del productor. - En relación al perfil del productor de sombreros de paja toquilla se constata que la mayoría son mujeres quienes realizan esta actividad, de tal modo que por cada 20 productores apenas uno es de sexo masculino.

El nivel educativo del artesano reviste situaciones preocupantes; debido a que, el 22,30 $\%$ de ésta población es analfabeta, y el 63,18 \% ha culminado únicamente la instrucción primaria, es decir muy pocos productores de sombreros han culminado la secundaria y menos aún la instrucción de nivel superior. Al parecer esta característica si bien no limita la actividad artesanal en su elaboración, si puede ser una barrera al momento de la determinación de costos, utilidad, comercialización, y en términos generales incrementos en la rentabilidad de esta actividad económica.

Nivel de instrucción del productor de sombreros de paja toquilla

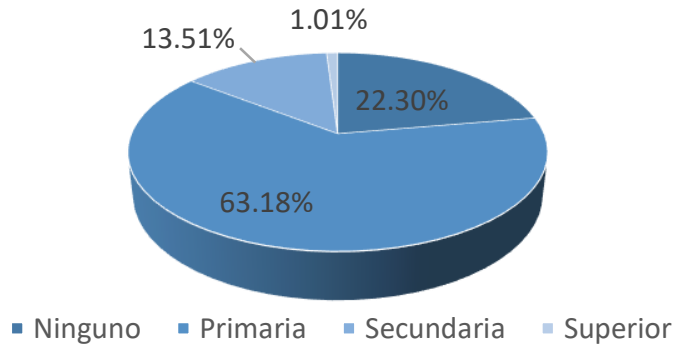

Figura 1. Nivel de instrucción de productor de sombreros de paja toquilla Fuente: Elaboración propia.

La edad del productor de sombreros muestra en su estructura gráfica que el desarrollo de ésta actividad inicia desde los diez años de edad y hay personas que la ejecutan hasta aproximadamente los 89 años. Sin embargo, el rango de mayor frecuencia de personas que practican esta labor se da entre los 35 y los 59 años de edad. De acuerdo a comentarios de los productores, a mayor edad mayores problemas en la elaboración de sombreros debido a problemas en la visión al ejecutar esta tarea.

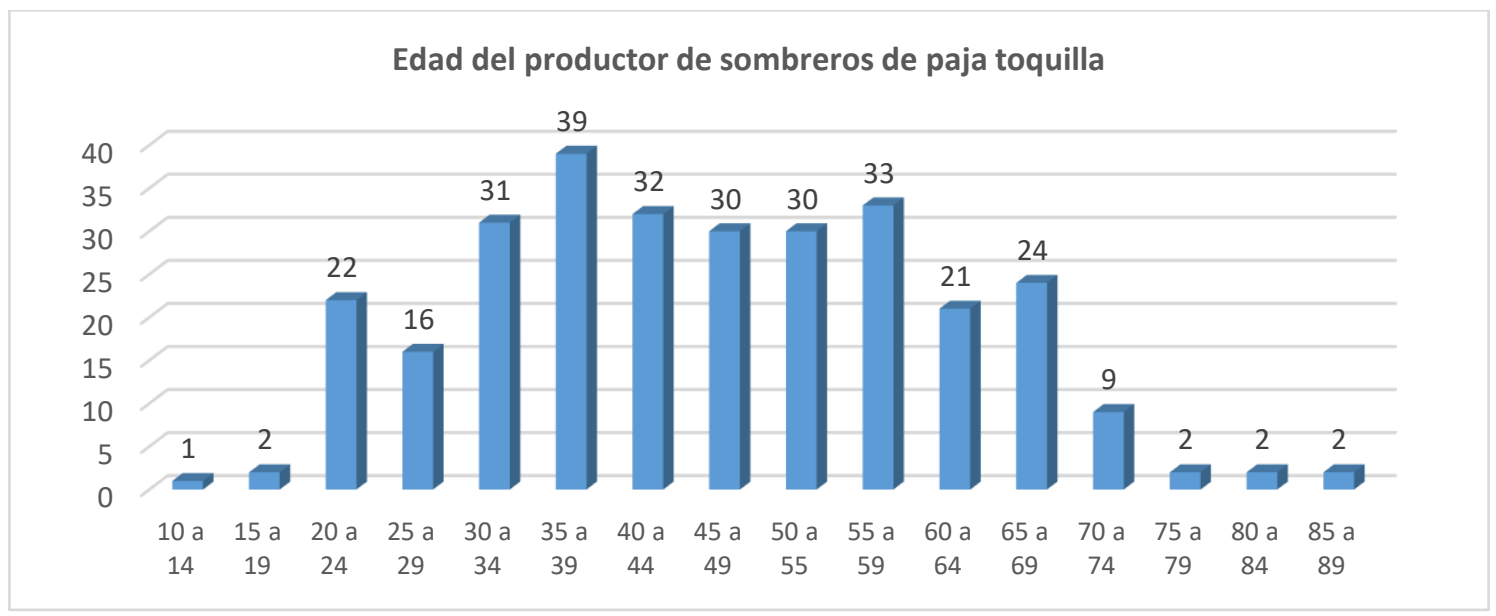

Figura 2. Edad del productor de sombreros de paja toquilla 
Fuente: Elaboración propia.

Característica de la producción. - La elaboración de sombreros en el cantón Sigsig se la realiza en los hogares. Para cuatro de cada cinco personas, es su única actividad económica. En consecuencia, una de cada cinco personas, desarrolla ésta actividad como complemento a la actividad económica principal que está vinculada a la agricultura y ganadería mayoritariamente.

En el mismo sentido, los productores que desarrollan esta actividad como su fuente principal de ingresos, le destinan varias horas de trabajo a esta actividad. Alrededor del $50 \%$ de los artesanos destinan entre cuatro y ocho horas por día a esta labor y el 17\% más de ocho horas de trabajo por día. Evidenciándose sobrecarga de trabajo para algunos productores.

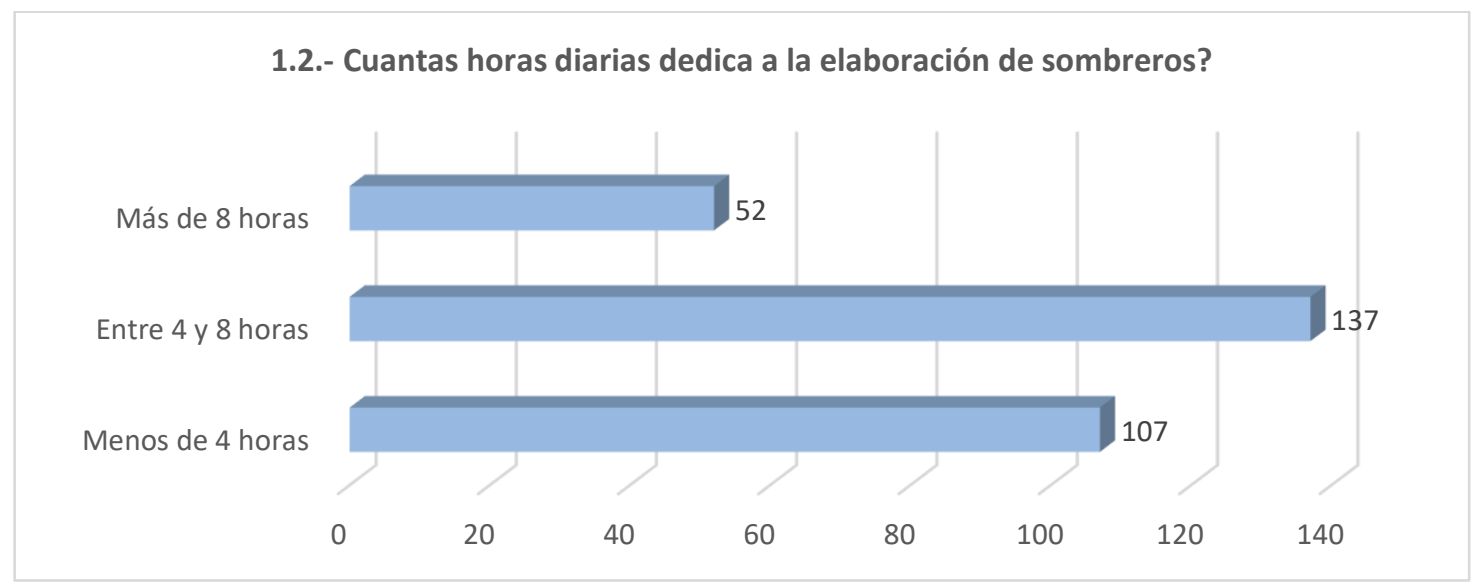

Figura 3. Horas diarias dedicadas a la elaboración de sombreros

Igualmente se ha consultado sobre la frecuencia de elaboración de los sombreros, siendo la respuesta que muestra mayor relevancia que las productoras desarrollan ésta actividad todos los días. En contraposición el $15 \%$ afirma desarrollar el tejido de sombreros "a veces". Esto en correspondencia con el hecho de que, en algunos hogares, es una actividad complementaria a la actividad económica principal.

1.3.- Frecuencia de elaboración de sombreros de paja toquilla

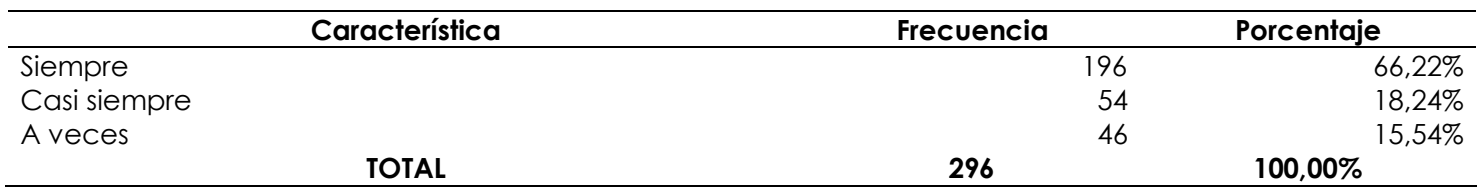

Figura 4. Frecuencia de elaboración de sombreros de paja toquilla

Fuente: Elaboración propia.

Si bien en la mayoría de hogares la elaboración de sombreros es la única actividad económica, es necesario acotar que no es la única persona que desarrolla esta actividad. 
En este sentido se ha consultado cuantas personas por hogar desarrollan esta actividad, cuyos datos van desde una persona, a 14 personas por hogar dedicadas a esta labor. Sin embargo, la mayor frecuencia de respuesta con 63,51\% es de una persona por hogar. Dos personas que trabajan en esta actividad representa el 19,59\% y tres personas el 8,78\%, cuyos datos son los que revisten mayor relevancia.

Finalmente, en este acápite se ha consultado si el Jefe de hogar elabora sombreros de paja toquilla, cuyo resultado muestra que una de cada cuatro personas si lo hace, mientras que la diferencia no lo realiza. Situación que ratifica que, si bien en estos hogares se desarrollan actividades vinculadas a la producción de sombreros del jefe de hogar o de uno de sus miembros, también se desarrollan actividades por parte de otros miembros del hogar que pueden ser o no jefes de familia, relacionadas con la producción agro productiva que contribuyen o complementan los ingresos del hogar.

\section{Volúmenes de producción y ventas}

Previo a conocer esta información, es necesario detallar los tipos de sombreros que se trabajan. En este sentido, se elaboran sombreros de variada calidad y precio. Los más relevantes son los siguientes: Chullado brisa, crochet, fino, llano, llanos pintados, modas y pariado. El tipo de sombrero que se elabora con mayor regularidad y que es el más común es el chullado brisa (81,76\% de los productores). Dicho esto, es necesario mencionar que conforme el tipo de sombrero varía el tipo de proceso de elaboración, en relación al tiempo requerido, en relación a la calidad debido al ancho de la hebra, al diseño y acabados.

Como se mencionó, el sombrero chullado es el sombrero que se confecciona con mayor frecuencia y que requiere menos tiempo para su elaboración. Se realizan en promedio 13 sombreros por mes; su precio de venta fluctúa entre los 6 y 7 dólares dependiendo de la calidad; su costo de producción es de aproximadamente 17 dólares y sus ventas varían entre los 70 y 80 dólares, en concordancia con la cantidad que se produce por mes.

El sombrero de tipo crochet, el llano, los llanos pintados y las modas, son sombreros que en términos de costos, precio y volumen de ventas se asemejan al sombrero chullado, por lo cual no se entra en demasiado detalle. Sin embargo, como se ha mencionado cada uno de ellos posee particularidades en su elaboración.

\begin{tabular}{ccccccc}
\hline \multicolumn{7}{c}{ Tipo de sombrero: Volúmenes de producción y ventas } \\
\hline Característica & $\begin{array}{c}\mathbf{2 . 2 . -} \text { Costo de } \\
\text { producción } \\
\text { por mes }\end{array}$ & $\begin{array}{c}\mathbf{2 . 3 . -} \text { Volumen } \\
\text { de Producción } \\
\text { al mes }\end{array}$ & $\begin{array}{c}\text { 2.6.- Precio de } \\
\text { venta unitario }\end{array}$ & $\begin{array}{c}\mathbf{2 . 5} \text { - Volumen } \\
\text { de ventas al } \\
\text { mes }\end{array}$ \\
\hline chullado(Brisa) & 18,08 & 13 & $\$$ & 6,42 & $\$$ & 75,62 \\
crochet & 17,93 & 12 & $\$$ & 6,28 & $\$$ & 71,67 \\
fino & 10,00 & 3 & $\$$ & 50,00 & $\$$ & 150,00 \\
llano & 18,15 & 13 & $\$$ & 6,43 & $\$$ & 75,98 \\
llanos pintados & 18,41 & 13 & $\$$ & 6,32 & $\$$ & 70,94 \\
\hline
\end{tabular}




\begin{tabular}{crrrrrr}
\hline modas & 19,09 & 13 & $\$$ & 6,89 & $\$$ & 75,53 \\
Pariado & 10,00 & 5 & $\$$ & 20,00 & $\$$ & 100,00 \\
\hline Promedio & $\mathbf{1 5 , 9 5}$ & $\mathbf{1 0}$ & $\mathbf{\$}$ & $\mathbf{1 4 , 6 2}$ & $\mathbf{\$}$ & $\mathbf{8 8 , 5 3}$ \\
\hline
\end{tabular}

Figura 5. Volúmenes de producción y ventas

Fuente: Elaboración propia.

Conforme la información obtenida, el sombrero pariado muestra que su precio de venta es superior a los mencionados con anterioridad ubicándose alrededor de los 20 dólares, se elaboran en promedio cinco por mes, su costo de producción en términos de materia prima es de diez dólares y sus ventas alcanzan a los 100 dólares por mes.

En contraposición, el sombrero fino lo realizan muy pocas personas, al ser un sombrero que requiere de mayor habilidad, destreza y tiempo continuo para su elaboración. Dependiendo del grado de finura se pueden elaborar hasta tres sombreros por mes, su costo de producción está alrededor de los diez dólares, su precio de venta es de 50 dólares, lo cual representa un volumen de ventas mensual de al menos 150 dólares.

Comercialización. - En relación a la comercialización de estos productos, ésta se realiza de diversas maneras. La más común es venderla en una plaza o mercado a un intermediario. Esta comercialización se la realiza regularmente cada fin de semana los días domingos desde las primeras horas de la mañana en el centro cantonal de Sigsig. En menor medida la venta de sombreros se la realiza desde el propio domicilio entregando a los comerciantes, esto sucede cuando el producto ha sido solicitado bajo pedido. También la comercialización es realizada por la asociación en el caso de las personas que son integrantes de la misma, lo cual posibilita que se pueda vender al mayorista evitando la intermediación.

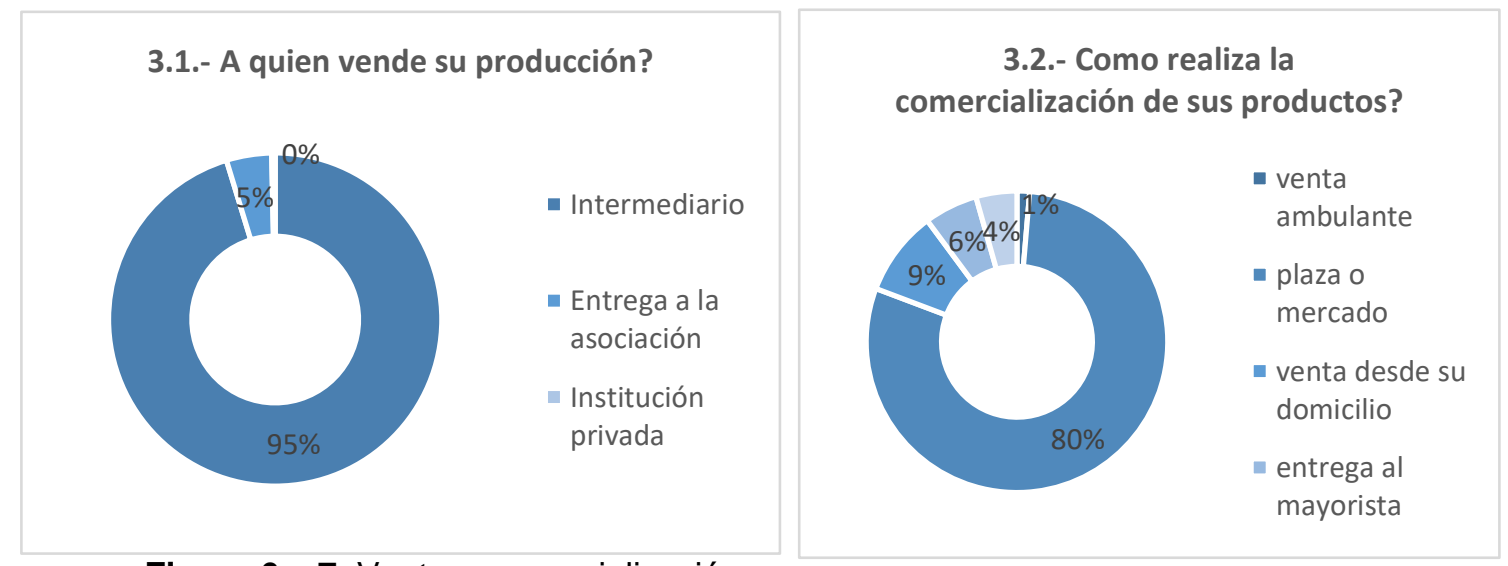

Figura 6 y 7 . Venta y comercialización

Fuente: Elaboración propia.

Si bien son diversas las formas de comercialización, gran parte de los casos, el producto intercambiado es un producto que no se encuentra terminado, situación que limita su margen de utilidad. Será necesario decir que la estrategia debe encaminarse en este sentido, debido a que en la cadena de valor el margen de utilidad de los productores de sombreros no es significativo, bajo la consideración del aporte de las personas en su 
calidad de productores, en cuanto a conocimiento, habilidad, acervo cultural y mano de obra.

\section{Asociatividad}

Conforme el levantamiento de información realizado, el 3,72\% de las personas encuestadas pertenecen a las dos asociaciones existentes dedicadas al tejido de sombreros de paja toquilla en el cantón. La Asociación María Auxiliadora y la Asociación TESYA, ubicadas en el centro cantonal. María Auxiliadora es la que conglomera mayor cantidad de socios con alrededor de cien personas. Entre los principales beneficios que consideran sus socios se destacan: la entrega de insumos, el apoyo en la comercialización y la capacitación. En relación a las obligaciones para con la asociación, está el cumplimiento con los plazos para entrega de los pedidos y la sujeción a las especificaciones técnicas en términos de tamaño y color requerido para la venta.

\section{Componentes de la estrategia}

Como parte preliminar a la definición de los componentes de la estrategia es necesario conocer cuáles son los nudos críticos que se presentan para los productores de sombreros al momento de la elaboración y comercialización de estos productos. En este sentido, al momento de la producción se requiere destinar tiempo de manera continua para la elaboración del sombrero, debido a que, al interrumpir el proceso, el tejido tiende a aflojarse y por tanto perder calidad.

A este problema se suma el vinculado al insumo principal que es la paja, misma que es susceptible a la humedad y que puede obscurecerse con el paso del tiempo perdiendo su color característico.

3.3.- Cuales han sido los nudos críticos o cuellos de botella en todo el proceso de producción y comercialización de los sobreros de paja toquilla.

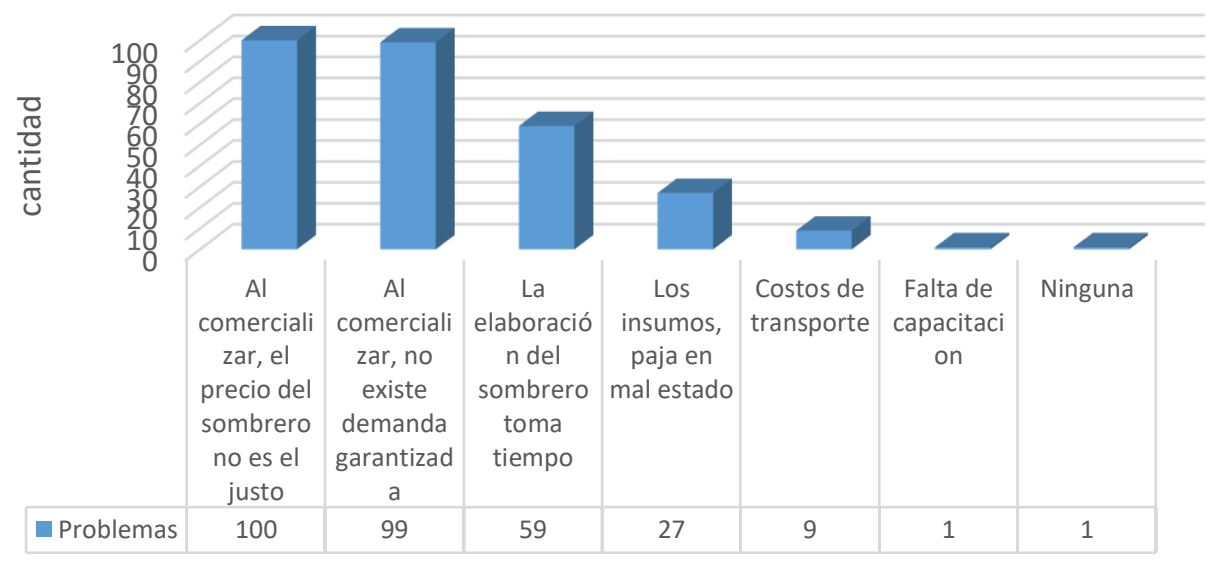

Figura 8. Nudos críticos en el proceso de producción y comercialización Fuente: Elaboración propia. 
También se ha consultado a las artesanas cual sería la posible estrategia para mejorar la producción de sombreros. Entre ellas se destacan -con mayor frecuencia de respuesta- el adquirir paja toquilla de calidad, que se pague un precio justo por el sombrero o que se exporte el sombrero desde el productor, también entre las respuestas destacables se requiere de procesos de capacitación en diseño, producción y terminados.

Si bien se registran más lineamientos, éstos no tienen un peso relevante en relación a la frecuencia de respuesta. Sin embargo, es importante mencionar que se requieren como posibles soluciones: adquirir máquinas para hormado de sombreros, implementar un lugar adecuado para la comercialización, mejorar la calidad del tejido, apoyo en la producción y comercialización por parte del Municipio y finalmente asociarse.

Desde la visión de los productores, la mayoría pone especial interés en las etapas del proceso productivo con diversidad de posibles estrategias y también analizan factores relacionados sobre la materia prima con relación a los costos y el lugar en donde se adquieren.

De las respuestas más relevantes de los encuestados el 45,61 \% considera que el precio es caro, el $14,53 \%$ lo considera como un precio normal y el $18,58 \%$ considera que es un producto de mala cantidad.

Finalmente, es importante decir que si lo que se busca es garantizar la demanda habrá que homogenizar los procesos para estandarizar los modelos conforme los requerimientos de los compradores. Mientras que, si la estrategia se vincula al ámbito turístico y cultural será necesaria potenciar la variabilidad en los diseños, texturas y colores no solo de los sombreros, sino de productos derivados de la paja toquilla.

\section{Visión desde la perspectiva Municipal}

Desde la visión del GAD cantonal se plantean otras reflexiones, como el hecho de que debe haber un enfoque de toda la cadena productiva del sombrero, en consideración de otros factores como la producción de la materia prima para su confección, que no ocurre en el cantón, las asociaciones que la procesan, el transporte desde su lugar de origen hasta el cantón, su comercialización y otros procesos y actores vinculados, hasta su llegada al productor final.

A partir del producto terminado, se da una fase de comercialización del sombrero, que mayoritariamente es hacia el exterior para su exportación. En algunos casos hay asociaciones de producción que hacen esa labor, pero la mayoría de productoras no están asociadas lo cual genera otros inconvenientes. 
Esta diversidad de actores y procesos que participan en la producción muestra que existe una gran cadena de problemas, relacionados de manera específica con la calidad del sombrero, que es demandado por un exigente mercado internacional al tratarse de un producto de consumo por parte de personas que tienen un estatus social importante en Europa y Estados Unidos, principalmente, para quienes la calidad cuenta.

Por tanto, aunque haya un buen tejido, si no hay una buena calidad que involucra materia prima, tamaño del sombrero, tipo de tejido, coloración de la paja, grado para el tejido, el sombrero se deprecia y podría caer su demanda en el mercado.

Sin embargo, las productoras mayoritariamente tejen sombreros cuatro o cinco a la semana en grado grueso, por así llamarlos, de menos complejidad en su elaboración y orientados a un mercado menos exigente, al alcance de un cliente local con menor poder adquisitivo.

Adicionalmente, es importante también el conocimiento y las oportunidades del mercado, la capacidad para poder exportar y para procesar, lo que genera inequidades para la cadena productiva. Son ciertos segmentos de la cadena los que tienen mayor beneficio cuando deberían ser aparentemente los productores los mayores beneficiarios en las tasas de retorno en la cadena de valor del sombrero, por lo que se vuelve necesario su revisión para viabilizar estrategias posibles.

Por lo tanto, se hace necesario emprender acciones para fortalecer a las productoras, su asociatividad y mejorar sus capacidades, articuladas con los distintos actores públicos y privados locales, que no ha sido efectivamente canalizada.

Hay un sin número de factores para tener un sombrero como producto final. Para conseguirlo debe haber pasado por una serie de eslabones productivos con diferentes actores en cada uno de ellos en una cadena productiva compleja, importante en las economías locales que debería ser abordado de manera integral.

En relación a los nudos críticos en la producción de sombreros, se menciona que la pandemia afectó mucho al sector dejándose de vender los sombreros mientras que la producción seguía realizándose. Hubo tejedoras que acumularon stocks de 100, 120, 150 sombreros, generándose situaciones de comercialización, organización, capacitación, fortalecimiento de las asociaciones de productores, entre otros.

Igualmente, el poder generar oportunidades de mercado propiciadas por las mismas productoras o las mismas asociaciones es un reto importante para que las productoras puedan ser las que se beneficien directamente. En la actualidad hay instituciones, organizaciones, empresas que se dedican a la exportación del sombrero y son los que mayores márgenes de ganancia tienen. Entonces poder vincular a las productoras campesinas, que están en las comunidades para que sean ellas las que hagan la producción, difusión, comercialización es importante para que pueda irse revirtiendo paulatinamente el beneficio, especialmente en sus ingresos. 
Se estima que el $90 \%$ de los sombreros se comercializan entre un 5 y 8 dólares, pero el precio final de esos sombreros en el consumidor final puede llegar a costar hasta unos $\$ 100$ en promedio. Si el productor participa en el $8 \%$ o $10 \%$ en el precio final, eso dice mucho de la vulnerabilidad que tienen los productores ante quienes participan como comerciantes, como procesadores, como gente que aparentemente es especializada en esos aspectos. Los mecanismos que permitan revertir esta situación evidentemente son retos que forman parte de esta estrategia de desarrollo.

En relación a la estrategia se menciona que debe enfocarse en la búsqueda de oportunidades de mercado tanto local, nacional como internacional, lamentablemente el mercado local se viene un poco a menos porque la gente no tiene la costumbre de utilizar sombrero en las urbes. Fomentar el uso del sombrero local y nacionalmente, podría ser un aspecto importante, además de informar cuales son los beneficios de utilizarlo.

En este escenario, el enfoque que se plantea desde el GAD es la difusión, por lo cual ha generado páginas web e impulsado ferias mundiales, como las planificadas en el presente año en EEUU y España, que debido a la pandemia no se realizaron, a fin de que sean las propias productoras las que vayan a exhibir los sombreros. Esta acción y los procesos de capacitación son los grandes retos en los que se encuentran inmersos en concordancia con sus competencias.

Para poder materializar ese objetivo, se ha planteado incluso presupuestos para participación en ferias nacionales e internacionales, con procesos paralelos de articulación institucional en consonancia con las atribuciones de los ministerios en el sector productivo. que lamentablemente no se ha sentido un apoyo que venga desde esos actores, como el ministerio de industria, comercio o el ministerio de turismo. Este último con el cual el GAD cantonal está potenciando el sector turístico integrando las artesanías como uno de los componentes que formen parte de una estrategia de generación de espacios físicos para la exhibición y comercialización de la misma.

En relación a como aporta esta actividad al desarrollo local, se menciona que existen aproximadamente, unas 1500 artesanas que producen cinco sombreros por semana, es decir veinte sombreros al mes. Se estaría hablando de 30.000 sombreros por mes y eso sería 240.000 sombreros por año.

La elaboración de sombreros aporta en el PIB local por lo menos en un millón de dólares al año, estos ingresos son generados por alrededor de 1500 familias dedicadas a esta actividad. Realizar 20 sombreros a $\$ 5$ equivaldría a unos $\$ 100$ mensuales por familia que generaría el sombrero. Considerando que en promedio existen cuatro miembros por hogar, serían 6000 personas involucradas, y esto representa el $25 \%$ de la población total del cantón. Evidentemente es una actividad fundamental, que moviliza varios actores y sectores, como el sector agro productivo, sector comercial, de transporte, entidades financieras, etc. 


\section{Definición de la estrategia}

Se ha considerado pertinente elaborar una matriz en la que se detallan los principales componentes, así como los principales actores involucrados en la actividad del tejido de sombreros de paja toquilla en el cantón.

\begin{tabular}{|c|c|c|c|}
\hline \multicolumn{4}{|c|}{ Matriz determinación de Estrategia } \\
\hline \multirow{2}{*}{$\begin{array}{l}\text { Principales } \\
\text { componentes }\end{array}$} & \multicolumn{3}{|c|}{ Principales actores } \\
\hline & $\begin{array}{l}\text { Productoras de } \\
\text { sombreros }\end{array}$ & $\begin{array}{l}\text { Asociaciones de } \\
\text { Toquilleras }\end{array}$ & GAD Municipal \\
\hline $\begin{array}{l}\text { Potencial de } \\
\text { desarrollo: } \\
\text { Elementos del } \\
\text { sistema económico } \\
\text { local }\end{array}$ & $\begin{array}{l}\text { Conocimiento y saber } \\
\text { ancestral del tejido de } \\
\text { sombrero }\end{array}$ & $\begin{array}{l}\text { Garantía de demanda } \\
\text { permanente de sombreros }\end{array}$ & $\begin{array}{l}\text { 1.- Agente de desarrollo } \\
\text { económico cantonal en el } \\
\text { marco de sus } \\
\text { competencias. } \\
\text { 2.- Decisión política de } \\
\text { incentivo a la actividad }\end{array}$ \\
\hline $\begin{array}{l}\text { Desarrollo de } \\
\text { capacidades y } \\
\text { competencias de los } \\
\text { actores }\end{array}$ & $\begin{array}{l}\text { 1.- Transferencia de } \\
\text { conocimiento de padres a } \\
\text { hijos. } \\
\text { 2.- Voluntad del } \\
\text { productor por capacitarse } \\
\text { permanentemente }\end{array}$ & $\begin{array}{l}\text { Gestión permanente con } \\
\text { instituciones públicas y } \\
\text { privadas para ejecutar } \\
\text { capacitación e innovación } \\
\text { en tejido, formas y } \\
\text { diseños de sombrero }\end{array}$ & $\begin{array}{l}\text { 1.- Articular con los } \\
\text { artesanos la ejecución de } \\
\text { capacitaciones, } \\
\text { propiciando espacios } \\
\text { adecuados para estos } \\
\text { eventos } \\
\text { 2.- Promocionar la } \\
\text { actividad del sombrero de } \\
\text { paja toquilla para evitar } \\
\text { su pérdida }\end{array}$ \\
\hline $\begin{array}{l}\text { Organización, } \\
\text { coordinación y } \\
\text { articulación entre } \\
\text { actores }\end{array}$ & $\begin{array}{l}\text { Asistir a reuniones de } \\
\text { coordinación y } \\
\text { manifestar sus problemas } \\
\text { así como predisponerse a } \\
\text { coordinar acciones } \\
\text { articuladas con otros } \\
\text { actores presentes en el } \\
\text { territorio que mejoren su } \\
\text { actividad económica }\end{array}$ & $\begin{array}{l}\text { Incentivar a los } \\
\text { productores individuales } \\
\text { a asociarse ofreciendo } \\
\text { beneficios que alienten a } \\
\text { disminuir los costos de } \\
\text { producción y mejoren sus } \\
\text { ventas priorizando el } \\
\text { beneficio colectivo sobre } \\
\text { el individual }\end{array}$ & $\begin{array}{l}\text { Firma de convenios de } \\
\text { cooperación entre los } \\
\text { distintos actores en } \\
\text { relación al fomento } \\
\text { productivo que propenda } \\
\text { al mejoramiento continuo } \\
\text { de ingresos de los } \\
\text { productores más } \\
\text { vulnerables }\end{array}$ \\
\hline $\begin{array}{l}\text { Mejoramiento } \\
\text { integral del proceso } \\
\text { productivo: } \\
\text { Capacidad } \\
\text { institucional, } \\
\text { Acervo tecnológico, } \\
\text { Infraestructura }\end{array}$ & $\begin{array}{l}\text { Estandarizar los procesos } \\
\text { productivos de } \\
\text { elaboración del sombrero, } \\
\text { así como los tamaños, } \\
\text { formas y colores para } \\
\text { garantizar productos de } \\
\text { calidad y mejorar los } \\
\text { precios de venta }\end{array}$ & $\begin{array}{l}\text { 1.- Adquisición de } \\
\text { maquinaria para hormado } \\
\text { de sombreros } \\
\text { 2.- Agilidad en los } \\
\text { procesos productivos } \\
\text { mediante la mecanización } \\
\text { en el proceso de } \\
\text { terminados }\end{array}$ & $\begin{array}{l}\text { 1.- Gestión para obtener } \\
\text { materia prima de calidad } \\
\text { y bajo costo } \\
\text { 2.- Articulación con } \\
\text { instituciones privadas } \\
\text { exportadoras para } \\
\text { garantizar la demanda de } \\
\text { manera permanente }\end{array}$ \\
\hline $\begin{array}{l}\text { Posicionamiento de } \\
\text { la marca y acceso a } \\
\text { mercados más } \\
\text { dinámicos }\end{array}$ & $\begin{array}{l}\text { Participar en ferias de } \\
\text { productores y en espacios } \\
\text { de promoción de la } \\
\text { actividad de sombrero de } \\
\text { paja toquilla }\end{array}$ & $\begin{array}{l}\text { Propiciar espacios de } \\
\text { participación de } \\
\text { productores asociados de } \\
\text { sombreros de paja } \\
\text { toquilla a nivel cantonal, } \\
\text { provincial y nacional. }\end{array}$ & $\begin{array}{l}\text { 1.- Crear ferias de } \\
\text { productores artesanales } \\
\text { 2.- Difundir el tejido de } \\
\text { sombrero de paja toquilla } \\
\text { como Patrimonio } \\
\text { Inmaterial de la } \\
\text { humanidad a nivel } \\
\text { nacional }\end{array}$ \\
\hline
\end{tabular}

Figura 9. Determinación de estrategia

Fuente: Elaboración propia. 
En este sentido la estrategia se encamina a generar oportunidades de mercado, diversificar y ampliar los canales de venta, mejorar los modelos y a la par mejorar la calidad, articular también la cadena productiva con los productores de materia prima, la integración de los actores que intervienen en el procesamiento del tejido del sombrero. Son los mayores retos que se deben afrontar y realizar.

\section{Conclusiones.}

- El sombrero de paja toquilla no es solamente el tejido, es una serie de otras actividades, por lo tanto es necesario realizar investigaciones adicionales que permitan tener un censo claro del número de productoras, número de producción semanal, mensual, anual, número de ingresos que genera el sombrero, quienes están interviniendo, cuales son los precios finales de venta en el exterior, cual es el componente o porcentaje que se quedan los productores, por lo tanto se requiere tener una línea integral para tomar decisiones estratégicas.

- Son múltiples las necesidades de los productores de sombreros de paja toquilla, sin embargo, la mayoría coincide en que los insumos son muy caros y el precio de venta del producto terminado es muy bajo, por lo tanto, es necesario buscar que el ingreso tenga un doble beneficio, eso sería un incentivo para que las nuevas generaciones se dediquen a esa actividad. Se puede ver que hay avances en las asociaciones que han diversificado las prendas y los productos lo cual viene de la mano de la capacitación, por lo que se pueden hacer productos complementarios al sombrero.

- Es un reto complejo cambiar el paradigma de las productoras de sombreros de paja toquilla sobre todo en las mujeres de edad avanzada, que realizan la actividad más por costumbre que como un trabajo que busca rentabilidad. La demanda del sombrero en la actualidad, requiere exigencias en relación a las formas, tamaños y productos cada vez de mejor calidad. La economía de subsistencia en este sector económico es altamente preponderante.

- El reto institucional es el de fomentar y sostener la actividad de tal modo que las nuevas generaciones vean en esta actividad, un atractivo que permita decir que, si bien van a seguir estudiando y superándose, pero también van a seguir tejiendo y por lo tanto seguir promoviendo el arte y la cultura.

- Desde la investigación de campo se evidenció que los sitios donde se realiza la comercialización de sombreros, es un lugar no apto para estos fines, dado que no se dispone de un mercado o una plaza específicamente desarrollada para esta actividad. Situación que provoca desorden, informalidad y cansancio en los productores al permanecer de pie. 


\section{Referencias bibliográficas.}

Acampora, T., Fonte, M. (2007). Productos típicos, estrategias de desarrollo rural y conocimiento local. Revista Opera, número 7, mayo 2007, pp.191-212 Universidad Externado de Colombia Bogotá, Colombia.

Aguirre. M., (2018). Las tejedoras de paja toquilla de la provincia del Azuay y los dilemas de la declaratoria del tejido como patrimonio inmaterial. Universidad Andina Simón Bolívar. pp. 1-111.

Alburquerque, F., (2008). Las estrategias de desarrollo económico territorial. Guía de aprendizaje sobre integración productiva y desarrollo económico territorial. pp. 346-362.

Anchundia-Rodríguez J., Andino-Chancay, T., Bailón-Cevallos, M, (2016). Producción y comercialización del sombrero de Paja toquilla en Montecristi. (Vol. 2, número 3). pp. 252-263.

Cortés, C., (2019). Estrategias de desarrollo rural en la UE: definición de espacio rural, ruralidad y desarrollo rural. Journal of chemical information and modeling, 53(9), pp.1-28.

Del Carpio, P., (2016). Estrategias mercadológicas e innovación en las artesanías una tradición transformadora. Poliantea, volumen xii, número 23, julio-diciembre, pp.77-110. Universidad de Guanajuato Campus Celaya- Salvatierra, México.

Durston J (2002), El capital social campesino en la gestión del desarrollo rural: díadas, equipos, puentes y escaleras. Comisión Económica para América Latina y el Caribe (CEPAL), pp.1-150. Santiago de Chile.

Hektor. (2005). Desarrollo Rural. Argonautas y caminantes. pp.87-99.

Kay, Cristóbal. (2007). Pobreza rural en América Latina: teorías y estrategias de desarrollo. Revista mexicana de sociología, 69(1), pp. 69-108.

López-Guzmán, T., Prada-Trigo, J., \& Pesantez, S. (2017). El patrimonio inmaterial de la humanidad como herramienta de promoción de un destino turístico. Estudios y Perspectivas en Turismo, volumen 26. pp.568-584.

Manquillo, N., La vulnerabilidad del sector artesanal colombiano en el ámbito nacional e internacional como 'negocio de conocimiento tradicional'. Estudio de caso: la denominación de origen artesanal 'Guacamayas' en el Acuerdo Comercial entre Colombia, Perú y la Unión Europea (2019). La Propiedad Inmaterial ํㅜ 28, Julio - diciembre 2019. pp. 167-209.

Moisá, Laura y Hernández, Adolfo (2010). El desarrollo rural de Medellín (Colombia) desde la perspectiva del desarrollo endógeno. Agronomía Colombiana, 28 (3). pp. 515-523.

Mora, J., \& Sumpsi, J. M. (2004). Desarrollo Rural: Nuevos Enfoques y perspectivas. Cuadernos FODEPAL. pp.1-42.

Ortiz, M., \& López, R. (2015). Caracterización socioeconómica de la comunidad artesanal de Nariño, Colombia. Lecturas de Economía - No. 82. Medellín, enerojunio. pp. 247-281. 
Palmitesta, L. (2001). Desarrollo local. Encuentro, año XXXIII (59). pp. 26-41.

Prada-Trigo, J., López-Guzman, T., Pesántez, S., \& Pérez-Gálvez, J. (2018). Turismo cultural, patrimonio inmaterial y elementos que inciden en la diferente satisfacción del visitante a partir del estudio del sombrero de pajatoquilla. Cuadernos De Turismo, Volumen 26. pp. 568 - 584.

Rojas, L., (2009). Guia de herramientas municipales para la promoción de desarrollo económico local/Fundación DEMUCA-San José, C.R. Primera edición. pp. 1-194.

Schejtman, A. (2010). Elementos para una renovación de las estrategias de desarrollo rural. Agronomía Colombiana 28(3). pp. 445-454. 


\section{PARA CITAR EL ARTÍCULO INDEXADO.}

Flores Aguilera, D. D., Uyaguari Quezada, J. J., \& Castillo Ortega, Y. (2021). Estrategia de desarrollo rural, una valoración desde las perspectivas endógenas en el caso de la producción de sombreros de paja toquilla, Sigsig-Azuay. ConcienciaDigital, 4(1.2), 6-28. https://doi.org/10.33262/concienciadigital.v4i1.2.1578

\section{Liencia}

El artículo que se publica es de exclusiva responsabilidad de los autores y no necesariamente reflejan el pensamiento de la Revista Ciencia Digital.

El artículo queda en propiedad de la revista y, por tanto, su publicación parcial y/o total en otro medio tiene que ser autorizado por el director de la Revista Ciencia Digital.
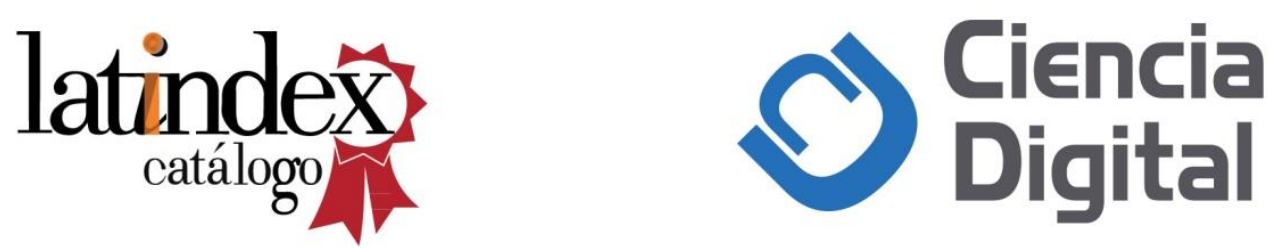